\title{
Adaptation of Esherichia coli to Antiobiotic Cycling via Single Nucleotide Polymorphisms
}

\author{
Samuel E. Hager , Ellen Jensen ${ }^{a}$, Timothy J. Johnson ${ }^{b}$ \& David Mitchell*a \\ ${ }^{a}$ Department of Biology, College of Saint Benedict/Saint John's University, Collegeville, MN \\ ${ }^{b}$ Department of Veterinary and Biomedical Sciences, University of Minnesota, St. Paul, MN \\ Student: samuelhager1@gmail.com \\ Mentor:dmitchell@,csbsju.edu*
}

\begin{abstract}
Bacteria are quick to adapt and evolve, especially under the effects of selective pressures from chemical antibiotics. In addition, bacteria may develop resistance to antibiotics from multiple classes simultaneously, making their eradication from the human body particularly challenging. This study aims to demonstrate that bacterial multiple-drug resistance can be developed and retained in a laboratory setting. Escherichia coli $\mathrm{B}$ was grown in tryptic soy broth in the presence of a small, increasing concentration of streptomycin. This exposure resulted in a strain of E. coli, which had an increased minimum inhibitory concentration (MIC) towards streptomycin, or "resistance." This resistant strain was then grown in like manner in nalidixic acid and then penicillin G. The result was a strain that became resistant to streptomycin and nalidixic acid, and increasingly resistant to nalidixic acid after penicillin G exposure. Additionally, the bacteria retained resistance to streptomycin and nalidixic acid even after exposure to those chemicals ceased. Genome sequencing and comparison to E. coli B reference strain REL606 revealed the emergence of point mutations with each exposure to an antibiotic. Of particular interest is a mutation associated with the appearance of nalidixic acid resistance. Base pair 4,553,488 was changed from adenine to guanine, resulting in a change from aspartate to glycine in the protein helicase. Previous studies have not indicated mutations to this locus as nalidixic acid resistance conferring. Thus, this mutation may be a novel mutation conferring $E$. coli B nalidixic acid resistance. Since the region of the mutated helicase is functionally undefined, a mechanism is not apparent. Further research needs to be done to confirm this hypothesis and illuminate a mechanism.
\end{abstract}

\section{KEYWORDS}

Bacteria; Escherichia coli; Evolution; Antibiotic Resistance; Nalidixic Acid; Streptomycin; Point Mutation; Single-nucleotide Polymorphism; Helicase; Minimum Inhibitory Concentration

\section{INTRODUCTION}

Competing microbes have been producing and reacting to antibiotics for thousands of years, and humans have also been exposed to these naturally occurring compounds for millennia. ${ }^{1}$ Even at the production of mass antibiotic synthesis in the $1940 \mathrm{~s}$, some microbiologists have suspected that bacteria might be able to inhibit antibiotics such as penicillin; today, multiple-drug resistant (MDR) strains pose a real and enduring threat to human health. ${ }^{1}$ The presence of these strains in hospitals and human waste watersheds, where antibiotic compounds abound, suggests that humans are responsible for pushing antibiotic-resistance alleles to fixation in certain microbial populations..$^{2-4}$ In the former, bacterial populations are exposed to a finite number of chemicals, providing a limited variety of sustained selective pressures. In the latter, bacteria are exposed to the same antibiotics being used in healthcare, as well as those being used for agriculture. In either scenario, these prolonged, varied selective pressures provide an environment where bacteria that adapt by developing resistance will out-compete other organisms. When a strain does this repeatedly in response to our most effective drugs, we are faced with a troubling situation, where a strain is resistant to many of our treatment options. Multiple drug resistant bacteria (for example, Carbapenem-resistant Enterobacteriaceae (CRE)) are a real and present threat to healthcare. ${ }^{5}$

Resistance to antibiotics is either innate or acquired. For example, bacteria such as E. coli are generally intrinsically resistant to vancomycin not because of any adaptation, but because of pre-existing traits that thwart vancomycin activity. ${ }^{6}$ Bacteria that do not possess intrinsic resistance to a given chemical may acquire resistance, either through de novo genetic mutations such as point mutations and chromosomal rearrangement (i.e., fluoroquinolone resistance through gyr $A$ mutations in E. coli), or through lateral gene transfer (i.e., acquisition of plasmids conferring resistance through possession of specific sets of genes). ${ }^{7}$ This study is concerned with the evolution of resistance to antibiotics via de novo genetic mutations.

This evolution may actually be expedited by human activity. Previous research demonstrated that baseline mutation rates are not constant, and may in fact be increased through exposure to antibiotics. ${ }^{8}$ In addition, numerous studies have begun to illuminate 
the importance of sub-lethal concentrations of antibiotics in the adaptation process, particularly in how they elicit the SOS response in bacteria. DNA damage, caused by antibiotics, radiation, or reactive oxygen species can activate the SOS response, which repairs damaged DNA. However, SOS is also associated with higher rates of polymerase errors, lateral gene transfer, and recombination, increasing the rate of resistance evolution. ${ }^{2,4,8}$ In addition, not only do sub-lethal concentrations encourage mutation and genetic variation, but they may also select for resistant bacteria.., 10 Thus, mutation and selection for resistance alleles may occur at sub-lethal concentrations of antibiotics. Stated simply, human use of antibiotics may actually contribute to the evolution of drug-resistant organisms, causing said drugs to become less effective.

A number of previous studies demonstrated that in vivo evolution occurs in response to antibiotic selective pressure via point mutations, ${ }^{11-14}$ however, fewer studies examined the sequential accumulation of resistance phenotypes. In this study, cultures were introduced sequentially to three different antibiotics at sub-lethal concentration in order to encourage high mutation rates. Mutation rates were not measured in this study, but by exposing a culture to an initial concentration below the lethal level, we hoped to decrease the time required for a resistance mutation to appear. Then, the antibiotic concentration was gradually increased until well over the wild-type minimum inhibitory concentration (MIC, the minimum concentration of antibiotic required to inhibit bacterial growth), in order to ensure resistance mutations were driven to fixation in the population. The process was repeated with each antibiotic, all from different classes, to investigate the possibility of the addition of new mutations and the retention of mutations no longer under selection pressure. This was a question worth investigation, since resistance mutations often confer a cost to the organism's fitness. ${ }^{13}$ In this study we show that mutations to bacterial genomes can confer resistance to antibiotics (i.e. an inheritable rise in the MIC) ${ }^{15}$ and remain in the genome, even in the absence of the mutation's particular selection pressure.

\section{MATERIALS AND METHODS}

\section{Cultures and materials}

All flasks were grown for about 24 hours at $30^{\circ} \mathrm{C}$ and $200 \mathrm{rpm}$ (New Brunswick Scientific C25 Incubator Shaker). Culture density was measured with the optical density at $600 \mathrm{~nm}$ ( $\mathrm{OD}_{600}$; Beckman DU640 spectrophotometer). Streptomycin sulfate salt (Sigma Chemical Co., CAS 3810-74-0), nalidixic acid sodium salt (Sigma Aldrich, CAS 3374-05-8), and penicillin G potassium salt (Nutritional Biochemicals Corp. CAS 113-98-4) were used to make stock solutions (4 mg/mL of the active compound) in sterile water. Tryptic soy agar (TSA) and tryptic soy broth (TSB) were used as media for growing plate and liquid cultures (BD Co.).

\section{Sequential exposure}

A pure culture of Escherichia coli B was streaked for isolation on TSA. A colony was selected for inoculation in $50 \mathrm{~mL}$ TSB and grown overnight, resulting in flask 0 . Then, $0.5 \mathrm{~mL}$ of flask 0 culture was transferred to $50 \mathrm{~mL}$ fresh TSB, which was prepared with streptomycin stock solution to yield a final streptomycin concentration of $0.53 \mu \mathrm{g} / \mathrm{mL}$ : flask 1 . After 24 hours, the $\mathrm{OD}_{600}$ was read and the next flask was prepared with the same 1:100 culture to TSB ratio, but with an increased concentration of streptomycin (Table 1). This was repeated seven times until a streptomycin concentration above the MIC (0.8 $\mathrm{kg} / \mathrm{mL}$, see Fig. 1) was reached.

\begin{tabular}{|c|c|c|c|c|c|c|c|c|}
\hline Flask & $\begin{array}{c}\text { Streptomycin } \\
\text { concentration } \\
(\boldsymbol{\mu g} / \mathbf{m L})\end{array}$ & $\mathbf{O D}_{\mathbf{6 0 0}}$ & Flask & $\begin{array}{c}\text { Nalidixic acid } \\
\text { concentration } \\
(\boldsymbol{\mu g} / \mathbf{m L})\end{array}$ & $\mathbf{O D}_{600}$ & Flask & $\begin{array}{c}\text { Penicillin G } \\
\text { concentration } \\
(\boldsymbol{\mu g} / \mathbf{m L})\end{array}$ & $\mathbf{O D}_{600}$ \\
\hline $\mathbf{1}$ & 0.53 & 1.60 & $\mathbf{7 . 1}$ & 0.01 & 1.69 & $\mathbf{7 . 5 . 1}$ & 1.60 & 1.06 \\
\hline $\mathbf{2}$ & 1.71 & 1.70 & $\mathbf{7 . 2}$ & 0.20 & 1.65 & $\mathbf{7 . 5 . 2}$ & 3.20 & 1.65 \\
\hline $\mathbf{3}$ & 7.11 & 1.48 & $\mathbf{7 . 3}$ & 0.79 & 1.49 & $\mathbf{7 . 5 . 3}$ & 9.90 & 1.91 \\
\hline $\mathbf{4}$ & 31.43 & 1.69 & $\mathbf{7 . 4}$ & 1.58 & 1.74 & & & \\
\hline $\mathbf{5}$ & 31.43 & 1.69 & $\mathbf{7 . 5}$ & 3.17 & 1.56 & & & \\
\hline $\mathbf{6}$ & 62.38 & 1.67 & & & & & & \\
\hline $\mathbf{7}$ & 122.84 & 1.68 & & & & & & \\
\hline
\end{tabular}

Table 1. E. coli B growth flasks with growth conditions and $\mathrm{OD}_{600}$ readings after approx. 24 hours. Note that the contents of flask 7 were used to "seed" flask 7.1 , which contained nalidixic acid. Thus the bacteria in 7.1 had been exposed to streptomycin, but were then removed from streptomycin to be exposed to nalidixic acid.

To prepare the next antibiotic treatment, a flask was set up with a 1:100 ratio of liquid culture from flask 7 to fresh TSB, as well as enough nalidixic acid stock solution to yield a sub-lethal concentration, resulting in flask 7.1. The process followed that of the streptomycin treatment, varying only in antibiotic concentrations and number of repeats. For the final treatment, two small well 
isolated colonies on a TSA streak plate of flask 7.5 were used as the inoculum and were placed into $100 \mathrm{~mL}$ TSB prepared with penicillin $\mathrm{G}$ stock solution, flask 7.5.1. The remainder of the procedure follows the aforementioned. Concentrations for all flasks and their $\mathrm{OD}_{600}$ readings after 24 hours are found in Table 1. Culture identity was confirmed through colony morphology and Gram staining.

\section{Resistance metric: $M I C$}

To measure the MICs of each antibiotic, 96-well plate 12 2-fold serial dilutions of antibiotics were made from TSB and antibiotic stock solutions. A sample from each selected liquid culture, an aliquot taken directly from the growth flasks of the evolution experiments, was diluted with water to a concentration of $1.66 \times 10^{8}$ colony forming units $(\mathrm{CFU}) / \mathrm{mL}$. Each well was filled with $300 \mu \mathrm{L}$ of a TSB-antibiotic dilution, and $3 \mu \mathrm{L}$ of the diluted liquid culture. Thus, the initial number of cells in each well was approximately $5 \times 10^{5} \mathrm{CFU}$. After incubating at room temperature for 20 hours, plates were stirred via brief shaking, and then read for $\mathrm{OD}_{600}$ values (Molecular Devices VersaMax microplate reader). Averaged raw absorbance values at $600 \mathrm{~nm}$ along with standard deviations from at least three replicates were graphed against antibiotic concentration.

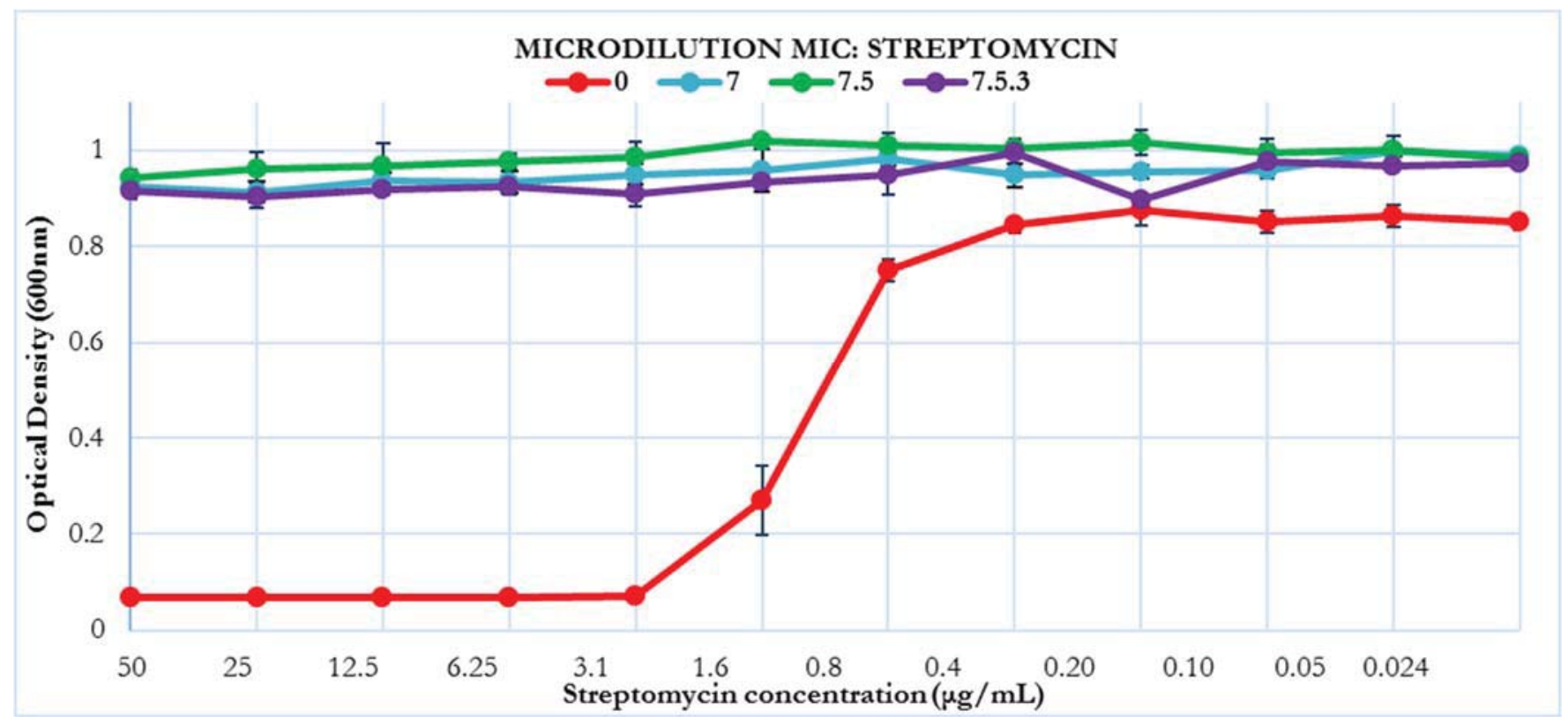

Figure 1. MIC for streptomycin, Series dilution in TSB, starting at $50 \mu \mathrm{g} / \mathrm{mL}$. Notice that the original parent strain in flask 0 has an MIC of about $0.8 \mu \mathrm{g} / \mathrm{mL}$. After exposure to streptomycin, this rises to more than $50 \mu \mathrm{g} / \mathrm{mL}$ (7, blue). This phenotype is retained by the cultures derived from 7 , but no longer exposed to streptomycin (7.5 and 7.5.3, green and purple, respectively).

\section{Genome Sequencing}

From the parent strain and each resistant strain, multiple isolated colonies were selected for genomic analyses via Illumina sequencing. Flask growths were grown overnight on MacConkey agar to check for visible contamination, then a streak of colonies from the plate was re-grown overnight in $2 \mathrm{~mL} \mathrm{LB}$ broth with shaking at $37^{\circ} \mathrm{C}$. DNA was extracted from overnight growths of each strain using the Qiagen DNEasy Kit. Library preparations were performed with Illumina reagents and DNA sequencing was performed at the University of Minnesota Genomics Center (Saint Paul, MN, USA). A minimum of 10 million high quality reads (Q30 average) was generated for each strain, corresponding to approximately 250-fold coverage or greater for each strain sequenced. Following quality filtering, reads were mapped to E. coli B strain REL606 (Genbank CP000819)15 using CLC Genomics Workbench version 9.0 (Qiagen) with a similarity fraction of 0.9 and minimum coverage of 100x for variant calling. Variants were called using CLC Genomics Workbench's Basic Variant Detection tool and outputted to tabular format. Variants were called at high minimum frequency $(>90 \%)$ to identify true variants relative to the reference genome and other sequenced strains in this study. Variants were also called at low minimum frequency $(<10 \%)$ to identify possible mixed populations within each growth containing a low-frequency mutation. Mutations unique to each strain sequenced, relative to the parent strain sequenced, were identified and annotated. The raw reads for this project are available in the NCBI BioProject PRJNA336011. 


\begin{tabular}{|c|c|c|c|c|c|c|c|}
\hline Sample & $\begin{array}{c}\text { Barcode } \\
\text { sequence }\end{array}$ & $\begin{array}{c}\text { \% Perfect } \\
\text { barcode }\end{array}$ & $\begin{array}{c}\text { \% One } \\
\text { mismatch } \\
\text { barcode }\end{array}$ & $\begin{array}{c}\text { Yield } \\
\text { (Mbases) }\end{array}$ & $\begin{array}{c}\text { \% clusters } \\
\text { passing filter }\end{array}$ & $\begin{array}{c}\text { \% } \geq \text { Q30 } \\
\text { bases }\end{array}$ & $\begin{array}{c}\text { Mean Quality } \\
\text { score }\end{array}$ \\
\hline Flask 0 & GATCAG & 98.66 & 1.34 & 16,264 & 95.44 & 92.74 & 36.17 \\
\hline Flask 7 & TAGCTT & 98.38 & 1.62 & 16,881 & 95.67 & 93.22 & 36.27 \\
\hline Flask 7.5 & GGCTAC & 98.17 & 1.83 & 16,672 & 95.11 & 92.13 & 36.04 \\
\hline Flask 7.5.3 & CTTGTA & 98.32 & 1.68 & 16,593 & 95.61 & 92.79 & 36.18 \\
\hline
\end{tabular}

Table 2. Illumina sequencing run statistics. Illumina defines their Quality Score $(\mathrm{Q})$ as $\mathrm{Q}=-10 \log _{10}(e)$.

\section{RESULTS AND DISCUSSION}

We found that the E. coli B liquid cultures consistently yielded OD readings above 1.5 in the presence of various antibiotics, even at levels far higher (>150-fold increase) than the initial, wild-type MIC (Table 1). This suggested that one or more mutations had in fact conferred antibiotic resistance to the surviving cells. There was a notable change in the MIC of a given antibiotic after exposure to that same antibiotic, displayed in Figures 1, 2 and 3, supporting the idea that there was some significant change in the bacteria after exposure to an antibiotic. In Figure 1, we noticed ancestral strain (flask 0) has an MIC of streptomycin of about $0.8 \mu \mathrm{g} / \mathrm{mL}$. After the stepwise streptomycin exposure, the resulting culture in flask 7 was able to grow in concentrations of streptomycin 150 times greater than the parent flask $0 \mathrm{MIC}$ of streptomycin. The E. coli cultures were found to grow to similar densities in high concentrations streptomycin, even after streptomycin exposure ceased (Fig. 1). This supports the idea that some resistance-associated mutations can occur during selection pressure and then be retained for many generations.

Likewise, in Figure 2, we noticed that cultures not treated with nalidixic acid have an MIC to nalidixic acid of about $0.2 \mu \mathrm{g} / \mathrm{mL}$. After exposure to nalidixic acid, the MIC rose to about $3.1 \mu \mathrm{g} / \mathrm{mL}$. This too was retained after exposure to nalidixic acid ceased (see Figure 2). Penicillin G exposure conferred a different result. In Figure 3 we see that all the flasks were resistant to penicillin at all levels. Since even the wild-type culture, flask 0, was unaffected, it is unclear whether exposure to penicillin G caused any change in the way the cell inhibits its function. However, in Figure 2 we observed that after being exposed to penicillin, the culture (7.5.3) seemed to have a higher MIC of nalidixic acid. Since MIC tests were performed using aliquots of the evolved cultures, it is possible that this is the result of an increased frequency of nalidixic acid-resistant mutants, and not a change in the DNA. Nevertheless, it plausible that exposure to one drug may give advantage against a completely different drug.

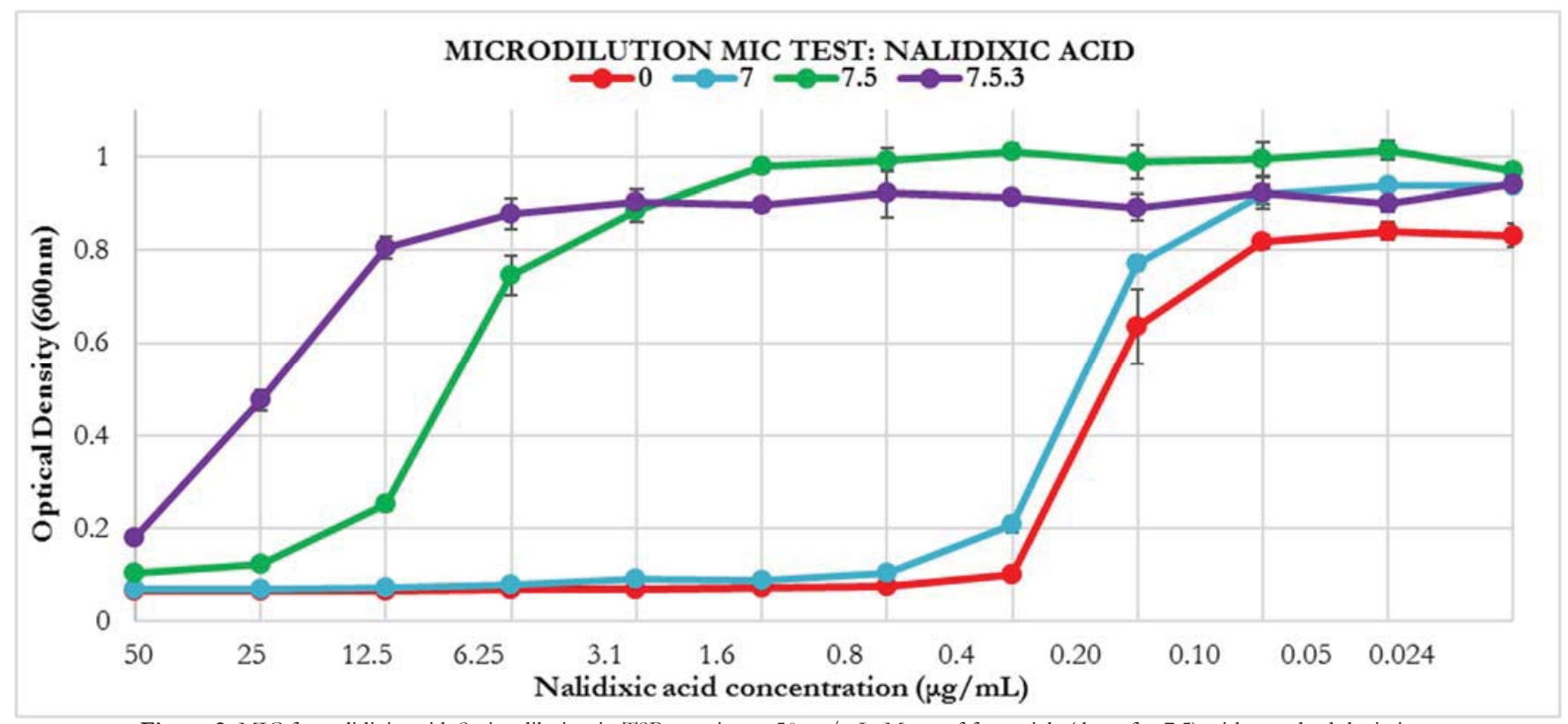

Figure 2. MIC for nalidixic acid. Series dilution in TSB, starting at $50 \mu \mathrm{g} / \mathrm{mL}$. Mean of four trials (three for 7.5) with standard deviation. 


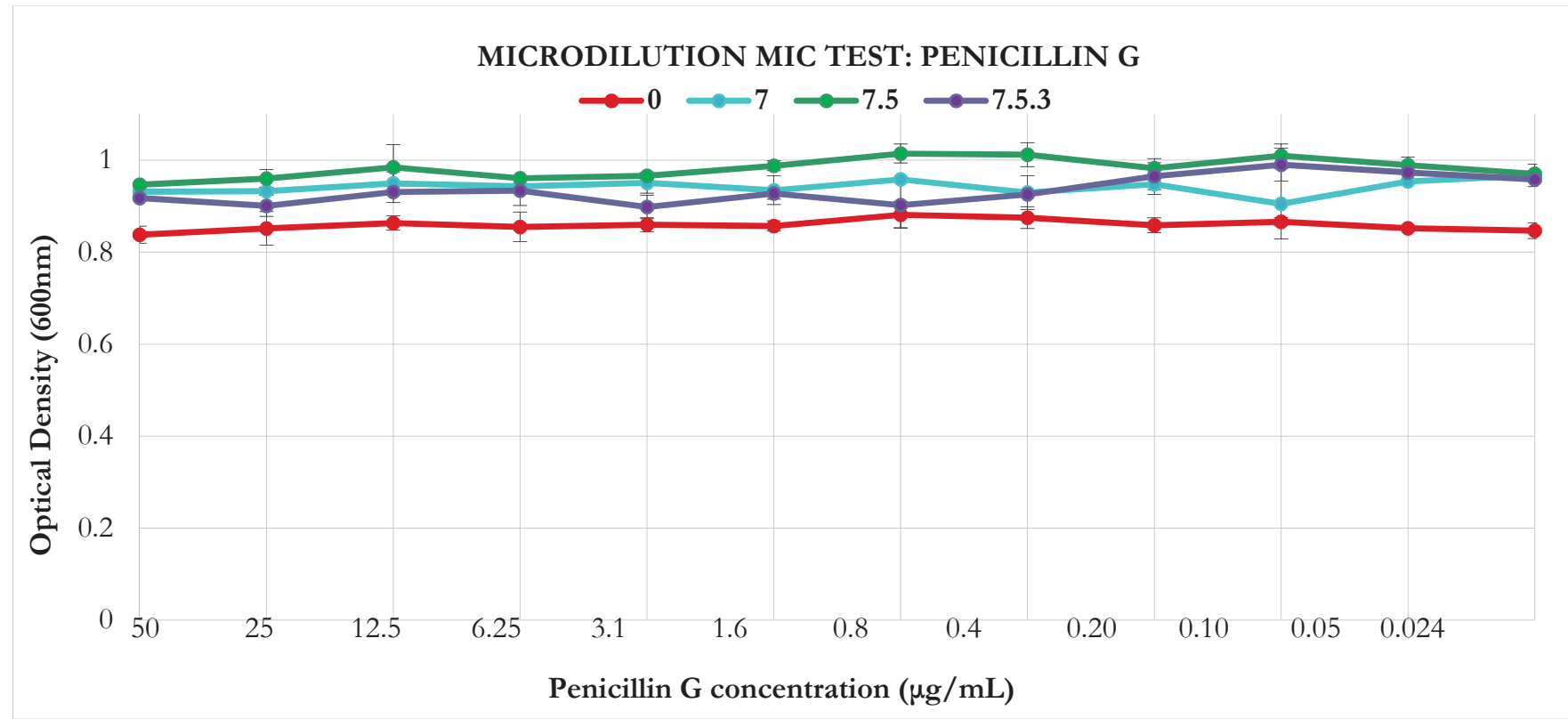

Figure 3. MIC for penicillin G. Series dilution in TSB, starting at $50 \mu \mathrm{g} / \mathrm{mL}$. Mean of four trials with standard deviation.

There existed the possibility that another species had contaminated and taken over the culture at some point, producing confounding results. However, this was put to rest with consistent Gram stain experiments indicating Gram-negative bacilli and colony morphology on EMB. The identity of all the samples as E. coli B was also confirmed with whole genome sequencing data, which provided details about how the bacteria adapted to this selection pressure.

Next-generation sequencing was performed for the four strains used for the MIC graphs. For all four cultures, SNP variants were called for strains with a minimum frequency of $90 \%$ with at least $100 \mathrm{x}$ mapping coverage. Table 3 contains a summary of the single-nucleotide polymorphisms among the samples meeting these criteria. Low-frequency SNP variants were also examined to determine if mutations existed in a potential mixed population within each strain examined. No evidence was found of low-level mutations or mixed populations via SNP analysis.

\begin{tabular}{|c|c|c|c|c|c|c|}
\hline \multirow{2}{*}{ Base pair } & \multicolumn{4}{|c|}{ Flask } & \multirow{2}{*}{ Mutation } & \multirow{2}{*}{ Gene } \\
\hline & 0 & 7 & 7.5 & 7.5.3 & & \\
\hline 77501 & $\Delta$ & & & & A to $G$ & Thiamine-binding periplasmic protein \\
\hline 456399 & $\Delta$ & $\Delta$ & & $\Delta$ & Deletion of C & Multidrug efflux RND transporter permease \\
\hline 3402195 & & $\Delta$ & $\Delta$ & $\Delta$ & $\mathrm{T}$ to $\mathrm{C}$ & 30 S ribosomal protein S12 (lysine 87 to glutamic acid) \\
\hline 4275010 & & & & $\Delta$ & G to $A$ & Intergenic repeat region \\
\hline 4529779 & & & $\Delta$ & $\Delta$ & $\mathrm{T}$ to $\mathrm{A}$ & Fimbrial protein FimH \\
\hline 4553488 & & & $\Delta$ & $\Delta$ & A to $G$ & Helicase (aspartate to glycine) \\
\hline 4553606 & & & $\Delta$ & $\Delta$ & $\mathrm{C}$ to $\mathrm{T}$ & Helicase (silent) \\
\hline
\end{tabular}

Table 3. Summary of single-nucleotide polymorphisms. A " $\Delta$ " indicates a particular culture $(0$, the original; 7 , exposed to streptomycin; 7.5 . exposed to streptomycin and nalidixic acid; 7.5.3, exposed to streptomycin, naalidixic acid, and penicillin $G$ ) differed from the REL606 reference sequence at the numbered base. The mutation column indicates the nucleotide change occurring. The gene column indicates the potential gene impacted and any amino acid differences.

\section{After streptomycin treatment}

After streptomycin treatment, base pair 77501 matches the reference-type (Table 3). This may be a function of fitness, or it may be a sequencing error or mixed culture in the parent strain leading to this observation. Whatever the case, it is unlikely that the protein impacted by this mutation (thiamine-binding periplasmic protein) plays a role in streptomycin resistance.

Base pair 3402195 in the S12 portion of the 30s ribosomal subunit mutated from T to C, changing lysine 87 to glutamic acid. This is likely the mutation that confers resistance to streptomycin, since aminoglycosides are known to bind to the 30 s ribosomal subunit. ${ }^{16}$ Since the mutation remained in the following samples, this would be an example of resistance via target modification. 
Chumpolkulwong et al. working with E. coli strain BL also observed streptomycin resistance when the mutation of lysine 87 to arginine in the $\mathrm{S} 12$ protein subunit occurred. ${ }^{17}$

\section{After nalidixic acid treatment}

After nalidixic acid treatment, base pair 456399 matches the reference type. In all the other samples, C is deleted from this site in the multidrug efflux RND transporter permease gene. While reversion is a possibility, other explanations seem more likely. A subpopulation within flask 7 that lacked the mutation may have fixed the reference type in flask 7.5 after the change in antibiotics. Considering this mutation causes a frameshift mutation in the predicted protein sequence of the permease, this mutation may be quite costly to the organism's fitness. These predicted fitness costs would support the explanation that a subpopulation drove the reference allele to fixation when the mutation was not advantageous.

Despite the inclusive-sounding name of the gene, it is doubtful that this alone could have led to nalidixic acid resistance, considering the deletion was present in flask 7.5.3, but the resistance that it would have conferred remained. No other mutation showing up in 7.5.3 would be able to explain the sustained MIC.

Base pair 4529779 mutated from $\mathrm{T}$ to $\mathrm{A}$ in the fimbrial protein fimH gene. While this mutation does fit the profile needed to support the MIC change — it changed and was fixed — the protein being affected is not related to the target of nalidixic acid, nor does it seem that fimbriae would assist in the metabolism or transportation of nalidixic acid molecules. However, it is worth investigating as a possible explanation, or partial explanation, for nalidixic acid resistance. One might confer only this allele to an organism, and observe any change in MIC of nalidixic acid.

Base pair 4553606 changed from $\mathrm{C}$ to $\mathrm{T}$ in helicase. While there is no change in amino acid sequence, we cannot deem this mutation irrelevant. A recent study indicates that interactions between chromatin and proteins, which may contribute to organism fitness, can be altered by non-synonymous mutations. ${ }^{18}$

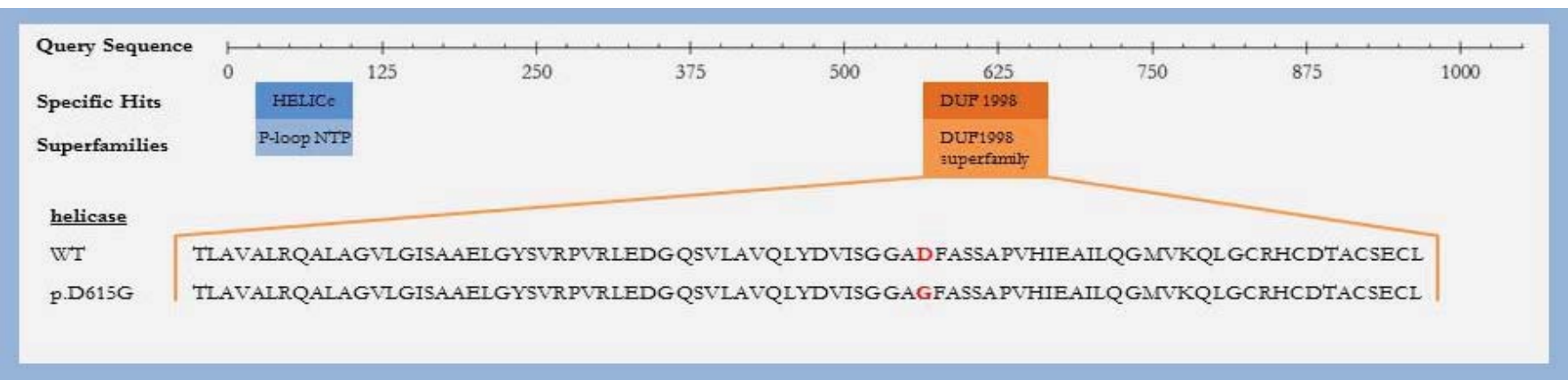

Figure 4. Conserved domains identified in the postulated helicase amino acid sequence (1051 amino acids). Multiple hits for "HELIc" occurred within the first 100 amino acid residues. DUF1998 spans residues 566 to 648 . The entire sequence of DUF is represented at the top and the mutation of interest is in yellow at 615. Figure created using data from BLAST and PSI-BLAST. ${ }^{19}$

Base pair 4553488 mutated from A to $\mathrm{G}$ in helicase, resulting in a change from aspartate to glycine. As seen in Figure 4, the mutation occurs in the middle of an amino acid sequence identified as Domain of Unknown Function (DUF)1998. ${ }^{19-20}$ DUF1998 is functionally uncharacterized, so it is unclear what the change from aspartate to glycine is doing to the function of the protein. Nevertheless, the mutation occurs in a predicted helicase, known to be involved in DNA replication, similar to gyrase and topoisomerase, the primary targets of quinolone activity. ${ }^{21}$

It's tempting to presume that some significant change is occurring. Since aspartate and glycine differ significantly in both size and charge, the protein may bend more freely because of the reduced steric interactions, or perhaps an allosteric binding site was changed. Also possible is a loss of a nalidixic acid binding site. Whatever the mechanism, this mutation is the most parsimonious explanation for resistance to nalidixic acid: the occurrence in the samples offers an explanation for changes in the MIC towards nalidixic acid (i.e., in Figure 2 and Table 3 we notice that the flasks which have this mutation also have an increased MIC towards nalidixic acid). In addition, this is a non-synonymous amino acid mutation, and it occurs in the category of cell activity that nalidixic acid targets: DNA unwinding. Thus, experiments are being planned to construct this chromosomal mutation.

\section{After penicillin G treatment}

After penicillin G treatment, base pair 456399, C was deleted, as it was in flask 0 and flask 7. Again, it is unclear precisely what this deletion does to the protein product, but it is probable that it is a disadvantageous frameshift mutation. In this case, it would seem that this mutation is not conferring additional nalidixic acid resistance, but damaging part of the protein structure. The only 
other mutation associated with penicillin treatment is found at 4275010, in an intergenic repeat region, which does not seem to promise much in the way of metabolic changes. MIC tests on a culture with this mutation and the absence of mutations 4275010 and 4553488 would indicate how this mutation relates to nalidixic acid.

\section{CONCLUSIONS}

E. coli are fast-growing, adaptable organisms. Small changes, even to one base pair out of four and a half million, can make a significant difference in cell phenotype. Under the right pressure, these changes can be highly beneficial for the bacterium, but not for humans; resistance of bacteria to antibiotics is a dangerous phenomenon. It is important that we understand the causes and mechanisms of antibiotic resistance in order to devise better treatments to infections and infectious diseases. Understanding how a bacterium may become resistant to nalidixic acid, for example, may lead us to a better understanding of fluoroquinoloneresistant infections, and what drug or drug combination may be used to keep up with fast-growing, highly adaptable bacteria.

\section{ACKNOWLEDGMENTS}

The authors thank Dr. Demelza (Koehn) Larson for her participation on his Honors thesis defense committee. All authors thank the College of Saint Benedict/Saint John's University and the University of Minnesota for their support of this project. The authors acknowledge the Minnesota Supercomputing Institute (MSI) at the University of Minnesota for providing resources that facilitated the research reported within this paper.

\section{REFERENCES}

1. Aminov, R. I. (2010) A Brief History of the Antibiotic Era: Lessons Learned and Challenges for the Future. Front. Micro. 1, 134.

2. Chow, L., Waldron, L., and Gillings, M.R. (2015) Potential impacts of aquatic pollutants: sub-clinical antibiotic concentrations induce genome changes and promote antibiotic resistance. Front. Micro. 6, 1-10.

3. Bollin, M., Jensen, E., and Mitchell, D. (2015) Occurrence of Multiple Antibiotic Resistant Bacteria in Aquatic Environments in Central Minnesota. Am. J. Undergrad. Res. 12, 19-35.

4. Gillings, M.R. and Stokes, H.W. (2012) Are humans increasing bacterial evolvability? Trends Ecol. Evol. 27, 346-352.

5. Van Duin, D., Kaye, K.S., Neuner, E.A., and Bonomo, R.A. (2013) Carbapenem-resistant Enterobacteriaceae: a review of treatment and outcomes. Diagn. Microbiol. Infect. Dis. 75, 115-120.

6. Alekshun, M.N and Levy, S.B. (2007) Molecular Mechanisms of Antibacterial Multidrug Resistance. Cell 128, 1037-1050.

7. Martinez, J.L. and Baquero, F. (2000) Mutation Frequencies and Antibiotic Resistance. Antimicro. Agents and Chemotherapy 44, 1771-1777.

8. Beaber, J.W., Hochhut, B., and Waldor, M.K. (2004) SOS response promotes horizontal dissemination of antibiotic resistance genes. Nature 427, 72-74.

9. Kaufmann, B.B. and Hung, D.T. (2010) The Fast Track to Multidrug Resistance. Mol. Cell 37, $297-298$.

10. Gullberg, E., Cao, S., Berg, O.G., Ilback, C., Sandegren, L., Hughes, D., and Andersson, D.I. (2011) Selection of Resistant Bacteria at Very Low Antibiotic Concentrations. PLoS Pathogens 7, e1002158.

11. Linkevicius, M.Sandegren, L., and Andersson, D.I. (2013) Mechanisms and fitness costs of tigecycline resistance in Escherichia coli. J. Antimicrob. Chemother. 68, 2809-2819.

12. Adler, M., Anjum, M., Andersson, D.I., and Sandegren, L. (2013) Influence of acquired $\beta$-lactamases on the evolution of spontaneous carbapenem resistance in Escherichia coli. J. Antimicrob. Chemother. 68, 51-59.

13. Levin, B.R., Perrot, V., and Walker, N. (2000) Compensatory mutations, antibiotic resistance and the population genetics of adaptive evolution in bacteria. Genetics 154, 985-997.

14. Cirz, R.T. and Romesberg, F.E. (2006) Induction and inhibition of ciprofloxacin resistance-conferring mutations in hypermutator bacteria. Antimicrob. Agents Chemother. 50, 220-225.

15. Escherichia coli B str. REL606, complete genome. NCBI ref seq: NC_012967.1. Accessed April 4, 2016. http://www.ncbi.nlm.nih.gov/nuccore/NC_012967.1

16. Carter, A.P., Clemons, W.M., Brodersen, D.E., Morgan-Warren, R.J., Wimberly, B.T., and Ramakrishnan, V. (2000) Functional insights from the structure of the $30 \mathrm{~S}$ ribosomal subunit and its interactions with antibiotics. Nature 407, $340-348$.

17. Chumpolkulwong, N., Hori-Takemoto, C., Hosaka, T., Inaoka, T., Kigawa, T., Shirouzu, M., Ochi, K., and Yokoyama, S. (2004) Effects of Escherichia coli ribosomal protein S12 mutations on cell-free protein synthesis. Eur. J. Biochem. $271,1127-1134$.

18. Bailey, S.D., Zhang, X., Desai, K., Aid, M., Corradin, O., Cowper-Sal Lari, R. Akhter-Zaidi, B., Scacheri, P.C., Haibe-Kains, B., and Lupien, M. (2015) ZNF143 provides sequence specificity to secure chromatin interactions at gene promoters. Nat. Comm. 2, 6186.

19. Altschul, S. F., Madden, T. L., Schäffer, A. A., Zhang, J., Zhang, Z., Miller, W., and Lipman, D. J. (1997) Gapped BLAST and PSI-BLAST: a new generation of protein database search programs, Nucleic Acids Res. 25, 3389-3402.

20. Marchler-Bauer A et al. (2015) CDD: NCBI's conserved domain database. Nucleic Acids Res. 43(Database issue): D222-226.

21. Aldred, K.J., Kerns, R.J., and Osheroff, N. (2014) Mechanism of quinolone action and resistance. Biochem. 53, $1565-1574$. 


\section{ABOUT THE STUDENT AUTHOR}

Samuel Hager graduated Magna Cum Laude and with distinction in his major from St. John's University in Collegeville, MN in 2016. He received a Bachelor of Arts with a major in Biochemistry and a minor in Theology. He began laboratory research for this paper with a 2015 Summer Biology Research Fellowship at St. John's University and the College of Saint Benedict.

\section{PRESS SUMMARY}

Bacteria have demonstrated the ability to evolve quickly in response to challenges from other organisms such as the use of antibiotics for agricultural and human consumption. Many bacteria are capable of transferring pieces of DNA between organisms as a rapid means of gaining resistance to antibiotics or some other competitive advantage. In this study we were interested to see if E. coli could overcome the administration of antibiotics by means of a chromosomal mutation that could then be passed on to all future generations of the bacteria. In addition, we were interested to see if such a mutation would remain if an organism were exposed to an antibiotic from a different class of antibiotic drugs. Our results indicate that such a means of gaining antibiotic resistance is possible and confirms previous work demonstrating how bacteria can strive in the presence of multiple antibiotics. 